\title{
Method of lower and upper functions and the existence of solutions to singular periodic problems for nonlinear differential equations of order two
}

Irena Rachưnková and Milan Tvrdý 


\title{
METHOD OF LOWER AND UPPER FUNCTIONS AND THE EXISTENCE OF SOLUTIONS TO SINGULAR PERIODIC PROBLEMS FOR NONLINEAR DIFFERENTIAL EQUATIONS OF ORDER TWO
}

\author{
IRENA RACHŮNKOVÁ \\ Department of Mathematics, Palacký University \\ Tomkova 40, 77900 Olomouc, Czech Republic \\ rachunko@matnw.upol.cz \\ Milan TVRDÝ \\ Mathematical Institute, Academy of Sciences of the Czech Republic \\ Žitná 25, 11567 Praha, Czech Republic \\ tvrdy@math.cas.cz
}

[Received May 26, 2000]

\begin{abstract}
We construct nonconstant lower and upper functions for the periodic boundary value problem $u^{\prime \prime}=f(t, u), u(0)=u(2 \pi), u^{\prime}(0)=u^{\prime}(2 \pi)$ and find their estimates. By means of these results we prove existence criteria for the problems $u^{\prime \prime} \pm g(u)=e(t), u(0)=$ $u(2 \pi), u^{\prime}(0)=u^{\prime}(2 \pi)$, where $\limsup _{x \rightarrow 0+} g(x)=\infty$ is allowed and $e \in \mathbb{L}[0,2 \pi]$ need not be essentially bounded.
\end{abstract}

Mathematical Subject Classification: 34B15, 34C25

Keywords: Second order nonlinear ordinary differential equation, periodic solution, singular problem, lower and upper functions, attractive and repulsive singularity, Duffing equation.

\section{Introduction}

In this paper we construct lower and upper functions to the periodic boundary value problem

$$
u^{\prime \prime}=f(t, u), \quad u(0)=u(2 \pi), \quad u^{\prime}(0)=u^{\prime}(2 \pi) .
$$

By means of these results, we prove existence criteria for the problems

$$
u^{\prime \prime} \pm g(u)=e(t), \quad u(0)=u(2 \pi), \quad u^{\prime}(0)=u^{\prime}(2 \pi),
$$

where $\lim \sup _{x \rightarrow 0+} g(x)=\infty$ is allowed and $e \in \mathbb{L}[0,2 \pi]$ need not be essentially bounded. We assume that $f:[0,2 \pi] \times \mathbb{R} \mapsto \mathbb{R}$ fulfills the Carathéodory conditions on $[0,2 \pi] \times \mathbb{R}$, i.e. $f$ has the following properties: (i) for each $x \in \mathbb{R}$ the function $f(., x)$ is measurable on $[0,2 \pi]$; (ii) for almost every $t \in[0,2 \pi]$ the function $f(t,$.$) is continuous$ on $\mathbb{R}$; (iii) for each compact set $\mathrm{K} \subset \mathbb{R}$ the function $m_{\mathrm{K}}(t)=\sup _{x \in \mathrm{K}}|f(t, x)|$ is Lebesgue integrable on $[0,2 \pi]$. 
For a given subinterval $J$ of $\mathbb{R}$ (possibly unbounded), $\mathbb{C}(J)$ denotes the set of functions continuous on $J$. Furthermore, $\mathbb{L}[0,2 \pi]$ stands for the set of functions Lebesgue integrable on $[0,2 \pi], \mathbb{L}_{2}[0,2 \pi]$ is the set of functions square Lebesgue integrable on $[0,2 \pi]$ and $\mathbb{A} \mathbb{C}[0,2 \pi]$ denotes the set of functions absolutely continuous on $[0,2 \pi]$. For $x$ bounded on $[0,2 \pi], y \in \mathbb{L}[0,2 \pi]$ and $z \in \mathbb{L}_{2}[0,2 \pi]$ we denote

$$
\begin{gathered}
\|x\|_{\mathbb{C}}=\sup _{t \in[0,2 \pi]}|x(t)|, \quad \bar{y}=\frac{1}{2 \pi} \int_{0}^{2 \pi} y(s) \mathrm{d} s \\
\|y\|_{1}=\int_{0}^{2 \pi}|y(t)| \mathrm{d} t \text { and }\|z\|_{2}=\left(\int_{0}^{2 \pi} z^{2}(t) \mathrm{d} t\right)^{\frac{1}{2}} .
\end{gathered}
$$

By a solution of $(1.1)$ we mean a function $u:[0,2 \pi] \mapsto \mathbb{R}$ such that $u^{\prime} \in \mathbb{A} \mathbb{C}[0,2 \pi]$, $u(0)=u(2 \pi), u^{\prime}(0)=u^{\prime}(2 \pi)$ and

$$
u^{\prime \prime}(t)=f(t, u(t)) \quad \text { for a.e. } t \in[0,2 \pi] .
$$

Definition 1. A function $\sigma_{1}$ is said to be a lower function of the problem (1.1) if $\sigma_{1}^{\prime} \in \mathbb{A} \mathbb{C}[0,2 \pi]$,

$$
\begin{gathered}
\sigma_{1}^{\prime \prime}(t) \geq f\left(t, \sigma_{1}(t)\right) \quad \text { for a.e. } t \in[0,2 \pi], \\
\sigma_{1}(0)=\sigma_{1}(2 \pi), \quad \sigma_{1}^{\prime}(0) \geq \sigma_{1}^{\prime}(2 \pi) .
\end{gathered}
$$

Similarly, a function $\sigma_{2}$ is said to be an upper function of the problem (1.1) if $\sigma_{2}^{\prime} \in \mathbb{A} \mathbb{C}[0,2 \pi]$

$$
\begin{gathered}
\sigma_{2}^{\prime \prime}(t) \leq f\left(t, \sigma_{2}(t)\right) \quad \text { for a.e. } t \in[0,2 \pi] \\
\sigma_{2}(0)=\sigma_{2}(2 \pi), \quad \sigma_{2}^{\prime}(0) \leq \sigma_{2}^{\prime}(2 \pi) .
\end{gathered}
$$

The lower and upper functions approach we will use here is based on the following theorem which is contained in [8, Theorems 4.1 and 4.2].

Theorem 2. Let $\sigma_{1}$ and $\sigma_{2}$ be a lower and an upper function of the problem (1.1), respectively.

(I) Suppose $\sigma_{1}(t) \leq \sigma_{2}(t)$ on $[0,2 \pi]$. Then there is a solution $u$ of the problem (1.1) such that $\sigma_{1}(t) \leq u(t) \leq \sigma_{2}(t)$ on $[0,2 \pi]$.

(II) Suppose $\sigma_{1}(t) \geq \sigma_{2}(t)$ on $[0,2 \pi]$ and there is $m \in \mathbb{L}[0,2 \pi]$ such that

$$
\begin{aligned}
f(t, x) \geq m(t) & \text { for a.e. } \quad t \in[0,2 \pi] \text { and all } x \in \mathbb{R} \\
\text { (or } f(t, x) \leq m(t) & \text { for a.e. } \quad t \in[0,2 \pi] \text { and all } x \in \mathbb{R} .)
\end{aligned}
$$

Then there is a solution $u$ of the problem (1.1) such that $\left\|u^{\prime}\right\|_{\mathbb{C}} \leq\|m\|_{1}$ and

$$
\sigma_{2}\left(t_{u}\right) \leq u\left(t_{u}\right) \leq \sigma_{1}\left(t_{u}\right) \text { for some } t_{u} \in[0,2 \pi]
$$




\section{Construction of lower and upper functions}

Proposition 1. Assume that there are $A \in \mathbb{R}$ and $b \in \mathbb{L}[0,2 \pi]$ such that

$$
\begin{aligned}
& \bar{b}=0, \\
& f(t, x) \leq b(t) \text { for a.e. } t \in[0,2 \pi] \text { and all } x \in[A, B],
\end{aligned}
$$

where

$$
B=A+\frac{\pi}{3}\|b\|_{1} .
$$

Then there exists a lower function $\sigma$ of the problem (1.1) such that

$$
A \leq \sigma(t) \leq B \text { on }[0,2 \pi] .
$$

Proof. Define

$$
\sigma_{0}(t)=c_{0}+\int_{0}^{2 \pi} g(t, s) b(s) \mathrm{d} s \text { for } t \in[0,2 \pi]
$$

where

$$
g(t, s)= \begin{cases}\frac{t(s-2 \pi)}{2 \pi} & \text { if } 0 \leq t \leq s \leq 2 \pi, \\ \frac{(t-2 \pi) s}{2 \pi} & \text { if } 0 \leq s<t \leq 2 \pi\end{cases}
$$

is the Green function of the problem $v^{\prime \prime}=0, v(0)=v(2 \pi)=0$ and

$$
c_{0}=-\frac{1}{2 \pi} \int_{0}^{2 \pi}\left(\int_{0}^{2 \pi} g(t, s) b(s) \mathrm{d} s\right) \mathrm{d} t .
$$

Then

$$
\sigma_{0}^{\prime \prime}(t)=b(t) \quad \text { a.e. on }[0,2 \pi]
$$

and

$$
\sigma_{0}(0)=\sigma_{0}(2 \pi)
$$

Furthermore, by virtue of (2.1) we have also

$$
\sigma_{0}^{\prime}(0)=\sigma_{0}^{\prime}(2 \pi) \text {. }
$$

Multiplying the relation (2.5) by $\sigma_{0}$, integrating it over $[0,2 \pi]$ and using the Hölder inequality we get

$$
\left\|\sigma_{0}^{\prime}\right\|_{2}^{2} \leq\|b\|_{1}\left\|\sigma_{0}\right\|_{\mathbb{C}}
$$

Further, as $\bar{\sigma}_{0}=0$, the Sobolev inequality (see [5, Proposition 1.3]) yields

$$
\left\|\sigma_{0}^{\prime}\right\|_{2}^{2} \leq \sqrt{\frac{\pi}{6}}\|b\|_{1}\left\|\sigma_{0}^{\prime}\right\|_{2},
$$


and so

$$
\left\|\sigma_{0}^{\prime}\right\|_{2} \leq \sqrt{\frac{\pi}{6}}\|b\|_{1},
$$

wherefrom using again the Sobolev inequality we get

$$
\left\|\sigma_{0}\right\|_{\mathbb{C}} \leq \frac{\pi}{6}\|b\|_{1} .
$$

Thus, the function $\sigma$ given by

$$
\sigma(t)=\frac{\pi}{6}\|b\|_{1}+A+\sigma_{0}(t) \text { for } t \in[0,2 \pi]
$$

satisfies (2.4). Furthermore, according to (2.1),(2.2) and (2.6)-(2.7) we have

$$
\sigma^{\prime \prime}(t)=\sigma_{0}^{\prime \prime}(t)=b(t) \geq f(t, \sigma(t)) \text { for a.e. } t \in[0,2 \pi]
$$

and

$$
\sigma(0)=\sigma(2 \pi), \quad \sigma^{\prime}(0)=\sigma^{\prime}(2 \pi),
$$

i.e. $\sigma$ is a lower function of (1.1).

The following assertion is dual to Proposition 1 and its proof will be omitted.

Proposition 2. Assume that there are $A \in \mathbb{R}$ and $b \in \mathbb{L}[0,2 \pi]$ such that

$$
\bar{b}=0
$$

and

$$
f(t, x) \geq b(t) \text { for a.e. } t \in[0,2 \pi] \text { and all } x \in[A, B]
$$

where $B$ is given by (2.3). Then there exists an upper function $\sigma$ of the problem (1.1) with the property (2.4).

\section{Applications to Lazer-Solimini singular problems}

In this section we will consider possible singular problems of the attractive type

$$
u^{\prime \prime}+g(u)=e(t), \quad u(0)=u(2 \pi), \quad u^{\prime}(0)=u^{\prime}(2 \pi)
$$

and of the repulsive type

$$
u^{\prime \prime}-g(u)=e(t), \quad u(0)=u(2 \pi), \quad u^{\prime}(0)=u^{\prime}(2 \pi),
$$

where

$$
g \in \mathbb{C}(0, \infty) \text { and } e \in \mathbb{L}[0,2 \pi]
$$

and it is allowed that $\lim \sup _{x \rightarrow 0+} g(x)=\infty$. 
The problem (3.1) has been studied by Lazer and Solimini in [6] for $e \in \mathbb{C}[0,2 \pi]$ and $g$ positive. In [9, Corollary 3.3], their existence result has been extended to $e \in$ $\mathbb{L}[0,2 \pi]$ essentially bounded from above. Here, we bring conditions for the existence of solutions to (3.1) without assuming boundedness of $e$.

Theorem 1. Assume(3.3) and let there exist $A_{1}, A_{2} \in(0, \infty)$ such that

$$
\begin{aligned}
& g(x) \geq \bar{e} \text { for all } x \in\left[A_{1}, B_{1}\right], \\
& g(x) \leq \bar{e} \text { for all } x \in\left[A_{2}, B_{2}\right],
\end{aligned}
$$

where

$$
B_{1}-A_{1}=B_{2}-A_{2}=\frac{\pi}{3}\|e-\bar{e}\|_{1}
$$

and $A_{2} \geq B_{1}$.

Then the problem (3.1) has a solution $u$ such that $A_{1} \leq u(t) \leq B_{2}$ on $[0,2 \pi]$.

Proof. Define, for a.e. $t \in[0,2 \pi]$,

$$
f(t, x)=e(t)-\left\{\begin{array}{cc}
g\left(A_{1}\right) & \text { if } x<A_{1} \\
g(x) & \text { if } x \geq A_{1} .
\end{array}\right.
$$

Then $f$ satisfies the Carathéodory conditions on $[0,2 \pi] \times \mathbb{R}$. Furthermore, by (3.4) and (3.6), $f$ satisfies (2.1)-(2.3) with $b(t)=e(t)-\bar{e}$ a.e. on $[0,2 \pi]$ and $[A, B]=$ $\left[A_{1}, B_{1}\right]$. Hence, by Proposition 1 there exists a lower function $\sigma_{1}$ of (1.1) such that $\sigma_{1}(t) \in\left[A_{1}, B_{1}\right]$ for all $t \in[0,2 \pi]$. Similarly, (3.5), (3.6) and Proposition 2 yield the existence of an upper function $\sigma_{2}$ of (1.1) such that $\sigma_{2}(t) \in\left[A_{2}, B_{2}\right]$ on $[0,2 \pi]$. Now, since $A_{2} \geq B_{1}$, we have $\sigma_{1}(t) \leq \sigma_{2}(t)$ on $[0,2 \pi]$ and the assertion (I) of Theorem 2 gives the existence of the desired solution $u$ to (1.1) which is naturaly also a solution to $(3.1)$.

Classical Lazer and Solimini's considerations [6] of the repulsive problem (3.2) have been extended by several authors (see e.g. [1]-[4], [7] and [10]). Here we present a related result, where $e$ need not be essentially bounded.

Theorem 2. Assume(3.3),

$$
\lim _{x \rightarrow 0+} \int_{x}^{1} g(\xi) \mathrm{d} \xi=\infty
$$

and

$$
g_{*}:=\inf _{x \in(0, \infty)} g(x)>-\infty .
$$

Furthermore, let there exist $A_{1}, A_{2} \in(0, \infty)$ such that

$$
\begin{aligned}
& g(x) \leq-\bar{e} \text { for all } x \in\left[A_{1}, B_{1}\right], \\
& g(x) \geq-\bar{e} \text { for all } x \in\left[A_{2}, B_{2}\right],
\end{aligned}
$$

where (3.6) is true and $A_{1} \geq B_{2}$.

Then the problem (3.2) has a positive solution. 
Proof. Denote

$$
K=\|e\|_{1}+\left|g_{*}\right|, \quad B=B_{1}+2 \pi K \text { and } K^{*}=K\|e\|_{1}+\int_{A_{2}}^{B}|g(x)| \mathrm{d} x .
$$

It follows from (3.7) that $\lim \sup _{x \rightarrow 0+} g(x)=\infty$ and there exists $\varepsilon \in\left(0, A_{2}\right)$ such that

$$
\int_{\varepsilon}^{A_{2}} g(x) \mathrm{d} x>K^{*} \text { and } g(\varepsilon)>0 .
$$

Define

$$
\widetilde{g}(x)= \begin{cases}g(x) & \text { if } x \geq \varepsilon \\ g(\varepsilon) & \text { if } x<\varepsilon\end{cases}
$$

and

$$
f(t, x)=e(t)+\widetilde{g}(x) \text { for a.e. } t \in[0,2 \pi] \text { and all } x \in \mathbb{R} \text {. }
$$

Now, we can argue as in the proof of Theorem 1 obtaining a lower function $\sigma_{1}$ and an upper function $\sigma_{2}$ of (1.1) such that $\sigma_{1}(t) \geq \sigma_{2}(t)$ on $[0,2 \pi]$. The assertion (II) of Theorem 2 (with $m(t)=g_{*}+e(t)$ a.e. on $[0,2 \pi]$ ) implies that (1.1) has a solution $u$ such that $u\left(t_{u}\right) \in\left[A_{2}, B_{1}\right]$ for some $t_{u} \in[0,2 \pi]$ and $\left\|u^{\prime}\right\|_{\mathbb{C}} \leq K$. It remains to show that $u(t) \geq \varepsilon$ holds on $[0,2 \pi]$.

Let $t_{0}$ and $t_{1} \in[0,2 \pi]$ be such that

$$
u\left(t_{0}\right)=\min _{t \in[0,2 \pi]} u(t) \text { and } u\left(t_{1}\right)=\max _{t \in[0,2 \pi]} u(t) .
$$

Clearly, $A_{2} \leq u\left(t_{1}\right) \leq B$. With respect to the periodic boundary conditions we have $u^{\prime}\left(t_{0}\right)=u^{\prime}\left(t_{1}\right)=0$. Now, multiplying the differential relation $u^{\prime \prime}(t)=e(t)+\widetilde{g}(u(t))$ by $u^{\prime}(t)$ and integrating over $\left[t_{0}, t_{1}\right]$ we get

$$
0=\int_{t_{0}}^{t_{1}} u^{\prime \prime}(t) u^{\prime}(t) \mathrm{d} t=\int_{t_{0}}^{t_{1}} e(t) u^{\prime}(t) \mathrm{d} t+\int_{t_{0}}^{t_{1}} \widetilde{g}(u(t)) u^{\prime}(t) \mathrm{d} t,
$$

i.e.

$$
\int_{u\left(t_{0}\right)}^{u\left(t_{1}\right)} \widetilde{g}(x) \mathrm{d} x=-\int_{t_{0}}^{t_{1}} e(t) u^{\prime}(t) \mathrm{d} t \leq K\|e\|_{1}
$$

Further,

$$
\int_{u\left(t_{0}\right)}^{A_{2}} \widetilde{g}(x) \mathrm{d} x \leq K\|e\|_{1}+\int_{A_{2}}^{B}|\widetilde{g}(x)| \mathrm{d} x=K^{*}
$$

which, with respect to (3.11), is possible only if $u\left(t_{0}\right) \geq \varepsilon$. Thus, $u$ is a solution to (3.2).

Example 3. Let $g(x)=\frac{1}{x^{\gamma}}$ on $(0, \infty)$. If $\gamma>0$, then Theorem 1 ensures the existence of a positive solution to (3.1) for any $e \in \mathbb{L}[0,2 \pi]$ such that

$$
\bar{e}>0 \text { and } \frac{\pi}{3} \bar{e}^{\frac{1}{\gamma}}\|e-\bar{e}\|_{\mathbb{L}}<1 .
$$


The function $e(t)=c+\frac{1}{\sqrt{2 \pi t}}-\frac{1}{\pi}$ with $c \in \mathbb{R}$ is not essentially bounded from above on $[0,2 \pi]$. However, it satisfies (3.12) if

$$
0<c<\left(\frac{3}{\pi}\right)^{\gamma} .
$$

We should mention that provided $e \in \mathbb{C}[0,2 \pi]$ or $e$ is essentially bounded from above, the condition $\bar{e}>0$ is sufficient for the existence of a solution to (3.1) (cf. [6] or [9], respectively).

Example 4 . Let $e \in \mathbb{L}[0,2 \pi]$ be essentially unbounded from below and let

$$
g(x)=\frac{1+\sin \left(\frac{\pi}{x}\right)}{x}-\arctan (x), \quad x \in(0, \infty) .
$$

Then $g$ verifies the assumptions (3.3), (3.7) and (3.8) of Theorem 2. Let us suppose that $\bar{e}=-5$. Then the equation $g(x)=5$ has exactly 5 roots in the interval $[0.125, \infty)$. In particular, we have (see Figures 1 and 2)

$$
\begin{aligned}
& x_{1} \approx 0.126804, x_{2} \approx 0.141071, x_{3} \approx 0.167853, x_{4} \approx 0.200541, x_{5} \approx 0.244461, \\
& g(x)>5 \text { on }\left(x_{2}, x_{3}\right) \cup\left(x_{4}, x_{5}\right) \text { and } g(x)<5 \text { on }\left(x_{1}, x_{2}\right) \cup\left(x_{3}, x_{4}\right) \cup\left(x_{5}, \infty\right) .
\end{aligned}
$$

Therefore, by Theorem 2, if

$$
\|e-\bar{e}\|_{\mathbb{L}} \leq \frac{3}{\pi}\left(x_{5}-x_{4}\right) \approx 0.0419392,
$$

the problem

$$
u^{\prime \prime}=\frac{1+\sin \left(\frac{\pi}{u}\right)}{u}-\arctan (u)+e(t), \quad u(0)=u(2 \pi), \quad u^{\prime}(0)=u^{\prime}(2 \pi)
$$

has a solution $u_{1}$ such that $u_{1}\left(t^{*}\right) \in\left[x_{4}, x_{5}+d_{1}\right]$ for some $t^{*} \in[0,2 \pi]$, where $d_{1}=$ $x_{5}-x_{4}$ (see Figure 3).

Similarly, by Theorems 1 and 2, if

$$
\|e-\bar{e}\|_{\mathbb{L}}<\frac{3}{2 \pi}\left(x_{5}-x_{4}\right) \approx 0.0209699,
$$

the problem (3.13) has at least 2 different solutions $u_{1}$ and $u_{2}$, where $u_{1}\left(t^{*}\right) \in\left(x_{5}-\right.$ $\left.d_{2}, x_{5}+d_{2}\right)$ for some $t^{*} \in[0,2 \pi]$ and $u_{2}(t) \in\left(x_{4}-d_{2}, x_{4}+d_{2}\right)$ for all $t \in[0,2 \pi]$, where $d_{2}=\frac{1}{2}\left(x_{5}-x_{4}\right)$ (see Figure 4$)$.

Finally, if

$$
\|e-\bar{e}\|_{\mathbb{L}} \leq \frac{3}{\pi}\left(x_{2}-x_{1}\right) \approx 0.0136238,
$$

the problem (3.13) has at least 3 different solutions $u_{1}, u_{2}$ and $u_{3}$, where $u_{1}\left(t^{*}\right) \in$ $\left[x_{5}-d_{3}, x_{5}+d_{3}\right]$ for some $t^{*} \in[0,2 \pi], u_{2}(t) \in\left[x_{4}-d_{3}, x_{4}+d_{3}\right]$ for all $t \in[0,2 \pi]$ and $u_{3}(t) \in\left[x_{1}, x_{2}\right]$ for all $t \in[0,2 \pi]$, where $d_{3}=x_{2}-x_{1}$ (see Figure 5 ). 


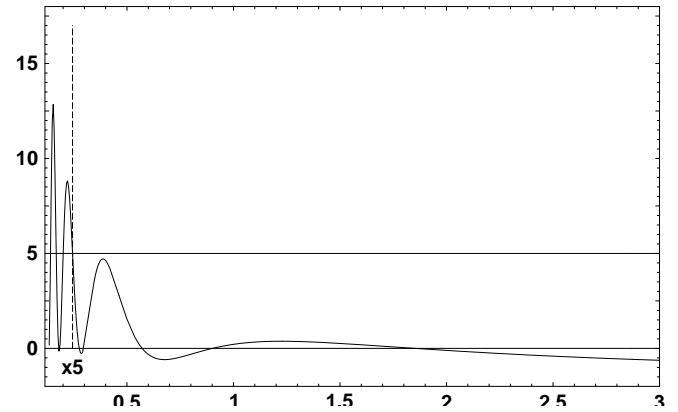

Figure 1.

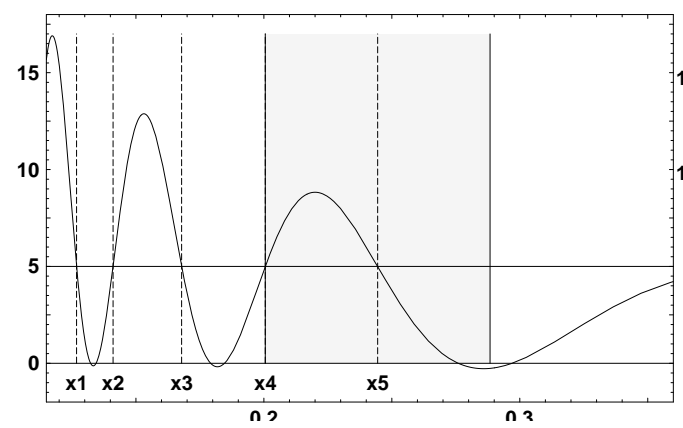

Figure 3.

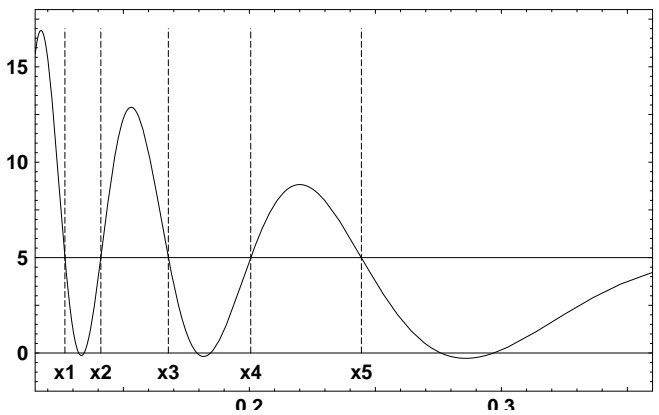

Figure 2.

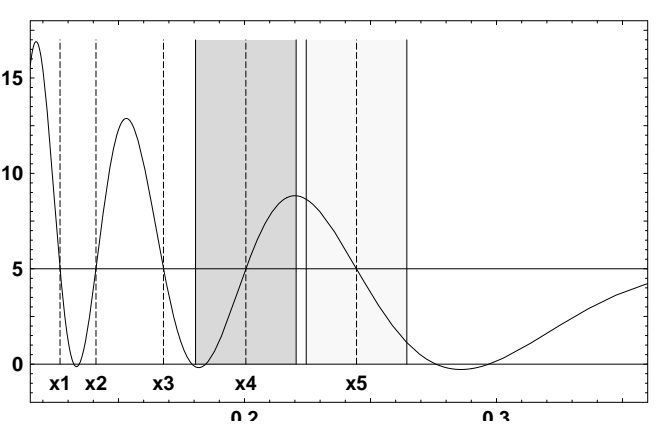

Figure 4.

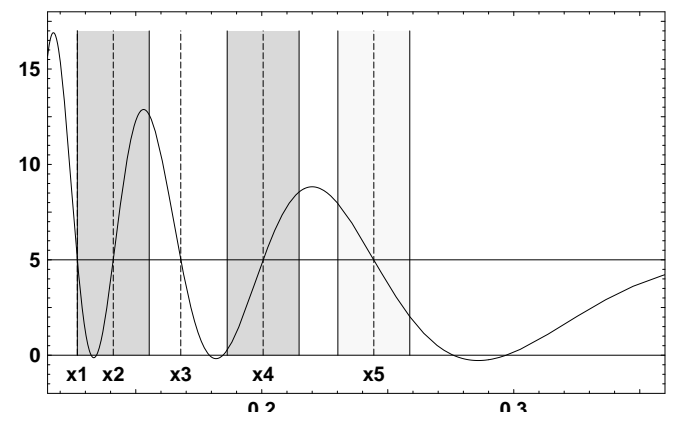

Figure 5. 
Acknowledgement: The support provided to I. Rachůnková by the grant No. 201/98/0318 of the Grant Agency of the Czech Republic and by the Council of Czech Government $\mathrm{J} 14 / 98: 153100011$ is gratefully acknowledged.

\section{REFERENCES}

[1] Del Pino, M., Manásevich, R. and Montero, A.: T-periodic solutions for some second order differential equations with singularities, Proc. Royal Soc. Edinburgh, 120A, (1992), 231-244.

[2] Fonda, A., Manásevich, R. and Zanolin, F.: Subharmonic solutions for some second-order differential equations with singularities, SIAM J. Math. Anal. 24, (1993), 1294-1311.

[3] Habets, P. and SAnchez, L.: Periodic solutions of some Liénard equations with singularities, Proc. Amer. Math. Soc. 109, (1990), 1035-1044.

[4] Mawhin, J.: Topological degree and boundary value problems for nonlinear differential equations, M. Furi (ed.) et al., Topological methods for ordinary differential equations. Lectures given at the 1st session of the Centro Internazionale Matematico Estivo (C.I.M.E.) held in Montecatini Terme, Italy, June 24-July 2, 1991. Berlin: SpringerVerlag, Lect. Notes Math. 1537, (1993), 74-142.

[5] Mawhin, J and Willem, M.: Critical Point Theory and Hamiltonian Systems, Applied Mathematical Sciences 74, Springer-Verlag, Berlin (1989).

[6] LAZER,A. C. and Solimini, S.: On periodic solutions of nonlinear differential equations with singularities, Proc. Amer. Math. Soc. 99, (1987), 109-114.

[7] Omari, P. and Ye, W.: Necessary and sufficient conditions for the existence of periodic solutions of second order ordinary differential equations with singular nonlinearities, Differential and Integral Equations 8, (1995), 1843-1858.

[8] Rachơnková, I. and TVRDÝ, M.: Nonlinear systems of differential inequalities and solvability of certain nonlinear second order boundary value problems, J. Inequal. Appl., to appear.

[9] Rachůnková, I., Tvrdý, M. and VRkoč, I.: Existence of nonnegative and nonpositive solutions for second order periodic boundary value problems, Preprint. Math. Inst. Acad. Sci. Czech Rep., 134/1999.

[10] Zhang, M.: A relationship between the periodic and the Dirichlet BVP's of singular differential equations, Proc. Royal Soc. Edinburgh, 128A, (1998), 1099-1114. 